\title{
SARS-COV2 Envelope Protein (E) interacts with the Lysophosphatidic Acid Receptor 1 (LPAR1) from humans
}

GIRISH NALLUR ( $\sim$ girish@vistarabio.com )

Vistara Bioscience LLC

\section{Research Article}

Keywords: SARS-COV2, Coronavirus, E protein, LPAR1, COVID Vaccine, COVID Treatment, Lung Fibrosis

Posted Date: July 17th, 2020

DOI: https://doi.org/10.21203/rs.3.rs-42592/v1

License: (c) (1) This work is licensed under a Creative Commons Attribution 4.0 International License.

Read Full License 


\section{Abstract}

A proteomic screen of human proteins interacting with the SARS-COV2 Envelope (E) protein identified LPAR1 as a strong candidate. Physical association of E protein and LPAR1 was confirmed by coimmunoprecipitation and cell surface staining. LPAR1-E protein interaction was confirmed in all eight human cell lines tested. Many additional proteins participating in the E protein interactions network were also enriched from each of the cell lines, some of which were cell type specific. These findings suggest that LPAR 1 is likely a cell surface receptor for the E protein, and pave the way for follow-on studies aimed at understanding the biological significance of the interactions in SARS-COV disease, including the signaling mechanisms.

\section{Introduction}

The Envelope (E) protein of SARS-COV2 is a small, transmembrane protein critical for virus life cycle. As a viroporin, it self-assembles in cell membranes forming pentameric protein-lipid pores to allow ion transport across cellular membranes. It has been suggested that interactions between the cytosolically oriented $\mathrm{C}$-terminal end of the $\mathrm{E}$ protein and the $\mathrm{C}$-terminal ends of the $\mathrm{M}$ or S proteins of SARS-COV2 are important for infection. Besides the channel activity, the E protein is also targeted to Golgi membranes and needed for virion morphogenesis and release. Despite these advances, the precise role of the $E$ protein in SARS-COV2 infection has remained enigmatic.

This report describes the in vitro protein interactions network of E protein in human cells. Proteomic analysis of a B cell line identified several cell surface receptor candidates, including one protein exhibiting a strong interaction with the $E$ protein. This protein was identified as LPAR1, a member of the $G$ protein coupled receptor family. LPAR1, and its interaction with the E protein, were confirmed by physical association of the $E$ protein to the cultured human cells, enriched by co-precipitation with $E$ protein, and co-localized with LPAR1 from each of seven additional cell lines of differing cell lineages.

LPAR1 is one of six Lysophospatidic acid receptors (LPAR1 - LPAR6) [1] encoded in the human genome, and a few additional LPAR-like proteins have been proposed. LPAR receptors contain transmembrane domains for ligand binding and signaling in human vascular, reproductive, nervous, and immune systems. LPA levels and LPAR receptor signaling mechanisms have been implicated in human airway disease, which is also a co-morbid condition in SARS-COV2 infection. However, it is not clear if the E protein interacts with cellular membrane components, involved in receptor signaling, or interferes with lipid metabolism to alter membrane structure or function. The potential for evaluating the $\mathrm{E}$ protein as a vaccine candidate and implications for SARS-COV disease therapy involving the E protein-LPAR1 signaling mechanisms are discussed.

\section{Results}


SARS-COV2 Envelope protein (E protein) interacts with human cellular proteins in a cell-type specific manner. To identify human proteins interacting with the E protein, several human cell lines were analyzed by Western blotting using a biotinylated, bacterial recombinant $E$ protein, and whole cell extracts from cultured cells [Fig. 1a]. A prominent band of around $42 \mathrm{kda}$ was observed in all four human samples tested. Several other bands were common between the cells, although the relative intensities of some varied from one cell type and another as well as with the $42 \mathrm{kda}$ band. A triplet of bands between $\sim 14$ to $\sim 17 \mathrm{kda}$ exhibited strong interactions with the E protein, and was unique to the $\mathrm{DOHH} 2$ (Diffuse large Bcell lymphoma line) sample. A control cell line, COS7, exhibited a pattern of interacting proteins different from any of the human cells. These observations suggest that the E protein interacts with multiple proteins from human cells, and that the interacting proteins vary with the cell type.

Protein interactions network of the E protein in DOHH2 cells. A STRINGseeker assay was performed as described previously [2], using the bacterially encoded E protein as bait and total cellular proteins from $\mathrm{DOHH} 2$ cells as preys. As shown in Fig1b, many proteins were enriched by the assay, including the $\sim 42$ kda protein shown in Fig. 1a. Barring minor alterations to protein mobilities of STRINGseeker products, there was good congruence with the pattern of bands observed by Western blotting and STRINGseeker purified proteins. Overall, the relative ratios of proteins in the STRINGseeker sample varied much less compared to the same in whole cell extracts, suggesting a degree of normalization of their concentrations. Network participation of the proteins and the required stoichiometry of binary interactions or complex formation within the networks are expected to promote normalization, which in turn will influence their enrichment.

\section{Identification of a candidate cell surface protein associating with the $\mathrm{E}$ protein in vitro. Preliminary} analysis of the E-STRINGseeker products from DOHH2 cells identified several cell surface proteins, suggesting that the E protein may possess a cell surface binding property. Further proteomic analysis of the sample showed that the most prominent among the cell surface proteins was Lysophosphatidic Acid Receptor 1 (LPAR1). The known molecular mass of LPAR1 correlated well with the $\sim 42 \mathrm{kda}$ band observed in Western blots.

LPAR1 is a clinical target in idiopathic pulmonary fibrosis, a chronic, progressive, and fatal fibrosing lung disease [3-7]. A member of the G-protein coupled receptors family, it is one of a family of receptors for Lysophosphatidic acid (LPA), mediating LPA signaling. LPAR1 contributes to the responses to tissue damage and infectious agents, with prominent roles in chemotaxis, cell migration, responses to injury, and pain perception, as well as triggers inflammation in response to bacterial lipopolysaccharide. LPAR1 has been shown to be expressed in many tissues, including abundantly in the brain and lung.

Binding of the E protein to the cell membrane is competed by LPAR1 antibody. A commercially available antibody to LPAR1 was employed for detecting LPAR1 by Western analysis in each of 8 human cell lines tested (Fig. 2a). The most prominent band was of $\sim 42 \mathrm{kda}$ protein, but 2 additional larger isoforms were variably present in different cell lines. In most cells other than DOHH2, LPAR1 was observed as a doublet of closely migrating species, which likely represent modified forms of the protein. Human AGTR1 and 
AGTR2 are also $42 \mathrm{kda}$ in size. The LPAR1 42kda protein co-migrates with AGTR2 (Fig. 2b), but is different from it since the larger bands in each lane exhibit different sizes as compared to those in Fig. 2a. Taken together, these observations confirm the expression of LPAR1 in all the cell lines tested.

The binding of the E protein to membrane associated LPAR1 was tested. The 8 human cell lines were grown to $90 \%$ confluence in 96 -well plates, fixed with formalin, and probed with a biotinylated $\mathrm{E}$ protein, with or without competition with unlabeled LPAR1 antibody. As shown in Fig. 3a., 7 of the 8 cell lines showed robust signals for cell surface binding of the E protein. The E protein binding signal was diminished by $10 \%$ to $43 \%$ in presence of 80 -fold molar excess of LPAR 1 antibody in different cell lines. HEK293 and HEK293T cells exhibited nearly 30\% reduction in signal intensities, and HeLa cells nearly $45 \%$. Surprisingly, 2 of the 4 lung cell lines, $\mathrm{H} 727$ and H460 showed only a modest inhibition by LPAR 1 antibody ( 11\%), while in a third line, H1581, LPAR1 antibody did not compete the binding of the $E$ protein. Taken together, the western and cell staining data demonstrate association of the E protein to cell surfaces, likely mediated by LPAR1.

Under the same conditions, the S protein probe showed negligible competition by LPAR1 antibody, although the signal intensities were significantly higher signal strengths (Fig. 3b). The higher signal strengths with the S protein may be ascribed to its association with AGTR1, AGTR2, or both. Intriguingly, cells which exhibited little or no inhibition of E protein binding by LPAR1 antibody, namely H460, H1581, and HepG2, exhibited modest inhibitions ( $9 \%$ to $14 \%$ ) with the S protein probe. The reasons for this are not clear. Perhaps, steric effects, collaboration with other membrane-bound receptors, membrane topology, or cell-specific modifications of LPAR1 may account for these differences.

The LPAR1 antibody was titrated over a 1,600-fold range for competition with the surface binding of the E protein to each of the 8 cell lines (Fig. 3d). All the cells, except HepG2, showed a dose response of inhibition of surface binding. E protein binding was reduced more than $60 \%$ in these cells at either the $400 x$ or $1600 x$ molar excess of LPAR1. The lack of inhibition with HepG2 cells is likely not due to absence of LPAR1 in these cells, but more so on account of its growth in multiple layers in culture, which may require different conditions for staining.

Co-purification and co-localization of LPAR1 with E interacting proteins: To confirm LPAR1 interaction with the E protein in vitro, STRINGseeker assays were performed with each of the 8 cells lines using the recombinant $E$ protein as bait. The interacting protein products were run alongside the corresponding whole cell extracts and probed by Western analysis. LPAR1 was detected using LPAR1 antibody (Fig. 4a) or co-localized with the $\sim 42 \mathrm{kda}$ protein using E protein as probe (Fig. $4 \mathrm{~b}$ ).

As shown in Fig. 4a., LPAR1 was detected in all 4 STRINGseeker samples tested, albeit with different degrees of enrichment. As with DOHH2 cells, $\mathrm{H} 1975$ and $\mathrm{H} 460$ cells showed substantial enrichments of LPAR1. LPAR1 recovery was lower in HEK293 or H1581 STRINGseeker samples, although the corresponding cell extracts showed abundant LPAR1 protein. The reasons for these are not clear. Perhaps, a competing interacting protein or cell-specific modifications of LPAR1 may affect complexing 
of LPAR1 with the E protein in the STRINGseeker assays. STRINGseeker products from the other 4 cell types were not analyzed.

Another set of samples was probed with the E protein (Fig. 4b). As observed previously with DOHH2, multiple interacting proteins were observed in the cell extracts and enriched by STRINGseeker, including, in particular, the $42 \mathrm{kda}$ band observed previously. The same band was detected in STRINGseeker products from DOHH2 cells, HEK293T, and HepG2, albeit with lower intensities in the latter two samples.

To investigate if LPAR1 also interacted with the S protein, STRINGseeker assays were performed with all 8 cells using the $S$ protein as bait, and analyzed as above. As shown in Fig. 4c., LPAR1 antibody failed to detect any band in the STRINGseeker products, nor did a $\sim 42$ kda protein co-purify with them. Many proteins ranging in size from $20 \mathrm{kda}$ to over $200 \mathrm{kda}$ were enriched in all STRINGseeker products with the $S$ protein (not shown); however, a protein co-migrating with LPAR1 was not one of them. The observations suggest that the interaction of LPAR1 is specific to the E protein.

\section{Conclusions}

This report describes the identification of the Lysophosphatidic acid receptor 1 (LPAR1) as an in vitro interacting protein of the SARS-COV2 Envelope (E) protein. While in the initial screen LPAR1 was highly enriched by interaction with the E protein using STRINGseeker, cells of hepatic, cervical, lung, and kidney origins also exhibit the E protein and LPAR1 interaction, suggesting it to be of wide occurrence in many human cell types.

Association of the E protein with cell membrane bound LPAR1 and co-purification with E protein interacting proteins from the eight cell lines tested strongly suggest that LPAR1 is an interactant of the E protein in humans. Heterogeneity of other proteins co-purifying with LPAR1 suggests the existence of multiple, cell-specific, signaling mechanisms and may bear some relevance to the multiple clinical manifestations of SARS-COV infections in humans. DOHH2, H1975, and H460 exhibited the strongest interactions while HEK293 and HEK293T cells showed noticeably lower recoveries.

It remains to be seen if any LPAR receptors other than LPAR1 was also enriched in the assays. However, the strong signature with LPAR1 suggests a key interaction with the E protein and LPAR1. Further studies with mapping the interacting regions of the two proteins can help characterize the interaction, and contributions from any other LPAR receptors. Mass spectrometry analysis of the STRINGseeker products from the 8 cell types can help identify any additional LPA receptors, or collaboration with other receptors [8].

Extension of these studies in SARS-COV infected cells can help understand the clinical significance of E protein interactions and further delineate molecular mechanisms of SARS-COV2 virus pathogenesis [9]. It remains to be determined if virus production is at all needed for the E protein association with LPAR1 in clinical samples, or if the protein, or its fragment, functioning as an exotoxin (such as Diphtheria toxin) is sufficient for clinical symptoms to occur, including lung abnormalities. Determining the detailed 
molecular signatures of the STRINGseeker products and assigning some of the proteins to well-studied signaling pathways may provide valuable clues, and a mechanistic response, of the $E$ protein associating with the LPAR1 protein network.

\section{Methods}

Cells: DOHH2 were purchased from Creative Biomart). HEK293, HEK293T, HepG2, HeLa, NCI-H1581, NCl$\mathrm{H} 460, \mathrm{NCl}-\mathrm{H} 727$ and $\mathrm{NCl}-\mathrm{H} 1975$ cell lines were purchased from ATCC. Cells were maintained in culture according to manufacturers' recommended protocols.

Sample preparation: Cells were grown to $80 \%$ to $90 \%$ confluence, scraped, and washed once with cold PBS. Approximately $4 \times 10^{6}$ cells were resuspended in 400 ul of $1 \times$ Cell Lysis buffer (9803, Cell Signaling Technology) and incubated at room temperature for $15 \mathrm{~min}$ with occasional mixing. Lysates were cleared by centrifugation and the supernatants stored in aliquots at $-20^{\circ} \mathrm{C}$. The protein concentrations of WCEs were determined at $280 \mathrm{~nm}$ with a NanoDrop Spectrophotometer.

Antibodies \& Reagents: Recombinant 2019-nCoV Envelope protein (RP01263LQ) was purchased from AbClonal Technology and recombinant SARS-COV2 Spike protein (Z03483) from GenScript. Mouse monoclonal antibody for LPAR1 was purchased from Santa Cruz Biotechnology; GAPDH was purchased from ProteinTech, and rabbit polyclonal antibody for AGTR2 was a kind gift from AbClonal.

Western blotting: 20 ug of total proteins WCE or 20 ul of STRINGseeker products were loaded per lane on a $4-20 \%$ gradient PAGE (GenScript) or $12 \%$ Bis-Tris gels (Invitrogen) under denaturing conditions. The proteins were transferred to Immobilon-FL transfer membranes (Merck Millipore Ltd.) for 2 hours at room temperature. Filters were washed once in TBS, blocked for $2 \mathrm{hr}$ in Intercept Blocking Solution (Li-Cor), and then incubated for 2 hours with biotinylated probe or antibody in Intercept Antibody diluent (Li-Cor) at a dilution of 1:2,000. After washing $3 x$ with 3 changes of PBST at room temperature, the filters were incubated for $1 \mathrm{hr}$ with 1:15,000 fold dilution of IR Dye 800 Streptavidin or IRDye ${ }^{\circledR}$ 680LT Goat antiMouse IgG Secondary Antibody (Li-Cor). Filters were washed with PBST and scanned in the Odyssey 9120 at $700 \mathrm{~nm}$ (Alexa) or $800 \mathrm{~nm}$ (Streptavidin). Images from Odyssey 9120 were analyzed with the Image Studio Lite software as per manufacturers protocols.

\section{Declarations}

Acknowledgements: The author acknowledges Vishwa Khatri, Swetha Tadepalli, and Crystal Harris for providing technical assistance, image analysis and quantitation. VK, ST, and $\mathrm{CH}$ were interns at Vistara Bioscience. They were support in part from a Technology Talent Bridge Grant from CTNext, which is gratefully acknowledged.

Competing interests: The author declares no competing interests.

\section{References}


1. Choi, JW et al. LPA Receptors: Subtypes and Biological Actions. Annu. Rev. Pharmacol. Toxicol. 50:157-186. 2010.

2. Nallur, GN. A proteomic model of SARS-COV2 infection by comparing the interactomes of BRD4 with BET-inhibition and SARS-COV2 viral proteins - implications for re-purposing approved drugs or ubiquitin-mediated degradation of select candidates. Research Square. DOI: 10.21203/rs.3.rs24573/v1. 2020.

3. Palmer, Scott M. et al. Randomized, Double-Blind, Placebo-Controlled, Phase 2 Trial of BMS-986020, a Lysophosphatidic Acid Receptor Antagonist for the Treatment of Idiopathic Pulmonary Fibrosis. CHEST, 154(5), 1061 - 1069. 2018.

4. Swaney JS, et al. A novel, orally active LPA(1) receptor antagonist inhibits lung fibrosis in the mouse bleomycin model. Br J Pharmacol. 160(7):1699-1713. 2010.

5. Olianas, MC et al. Antidepressants induce profibrotic responses via the lysophosphatidic acid receptor LPA1. J. Pharmacol. 873(172963). 2020.

6. Nishikawa, $\mathrm{N}$ et al. Therapeutic efficacy of a novel LPA1 receptor antagonist, UD-009, in a bleomycininduced pulmonary fibrosis model. Eur. Res. J. 48: PA4032; DOI: 10.1183/13993003. 2016.

7. Tager, AM et al. The lysophosphatidic acid receptor LPA1 links pulmonary fibrosis to lung injury by mediating fibroblast recruitment and vascular leak. Nature Med. 4 [1], 45 -54. 2008.

8. Teoh CM, et al. Integrin and GPCR Crosstalk in the Regulation of ASM Contraction Signaling in Asthma. J Allergy (Cairo). 2012;2012:341282.

9. Ruch, TR and Machamer, CE. The Coronavirus E Protein: Assembly and Beyond. Viruses, 4, 363-382. 2012.

\section{Figures}


a.

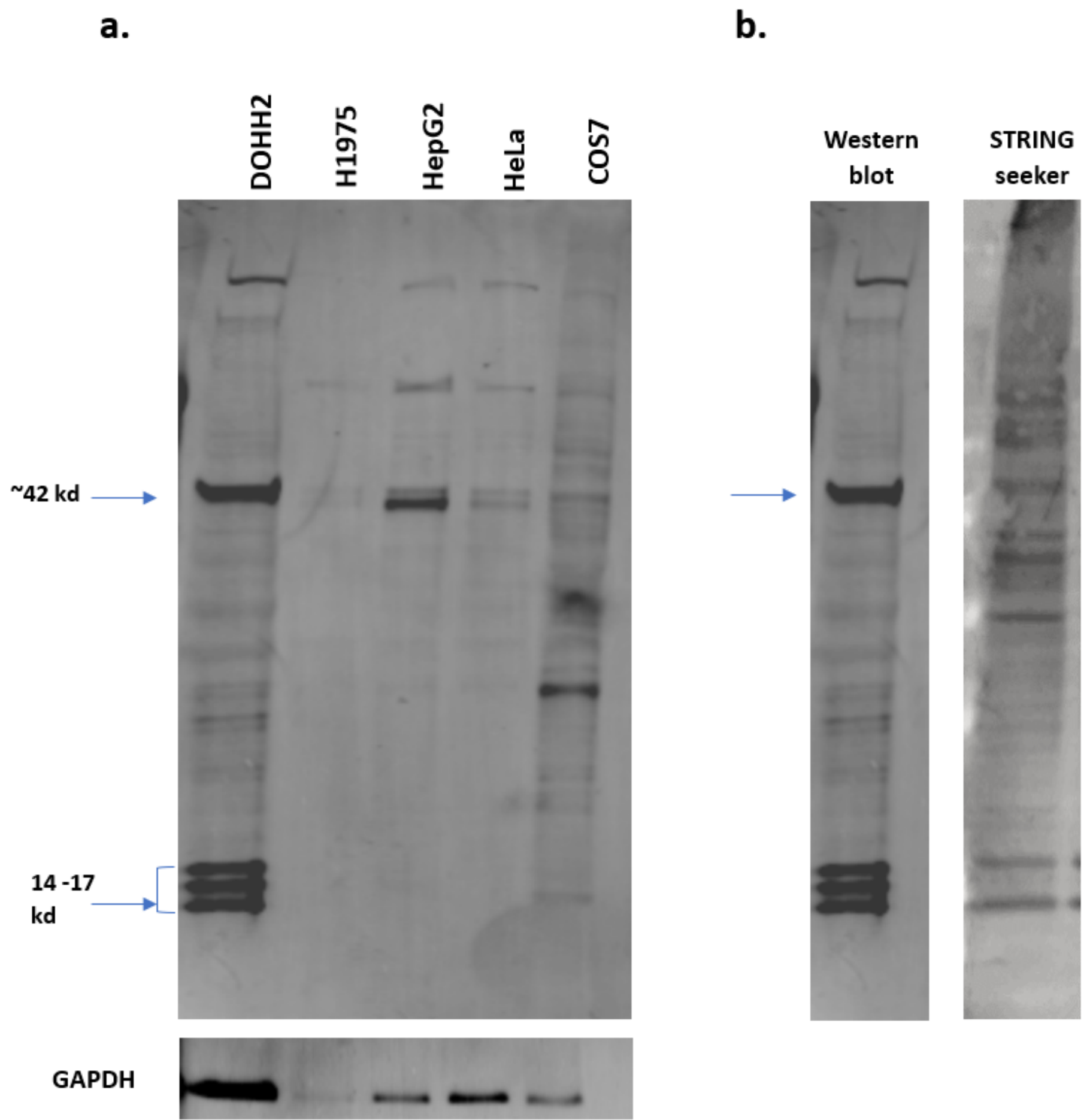

b.

Figure 1

E protein interacts with human cellular proteins in a cell-type specific manner. 


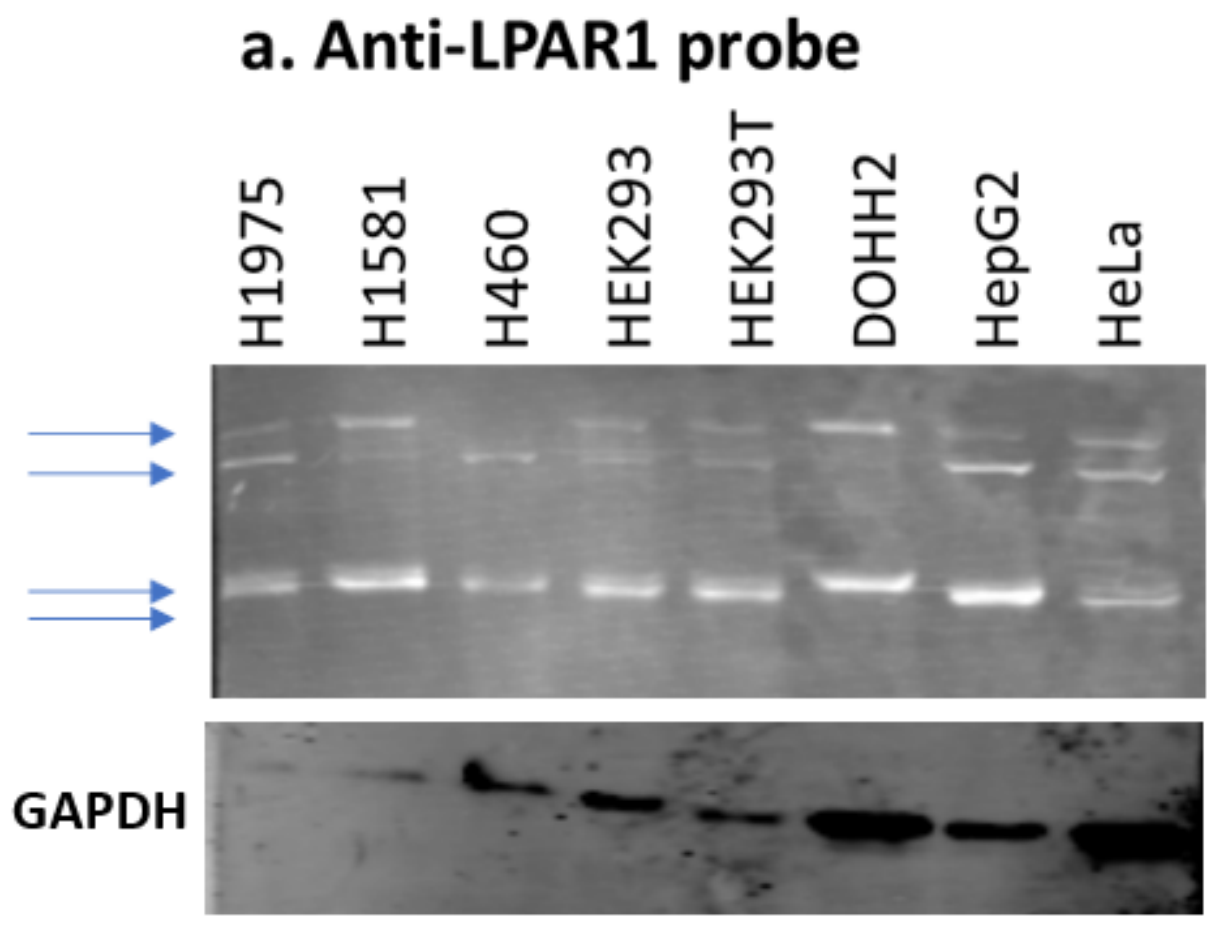

b. Anti-AGTR2 probe

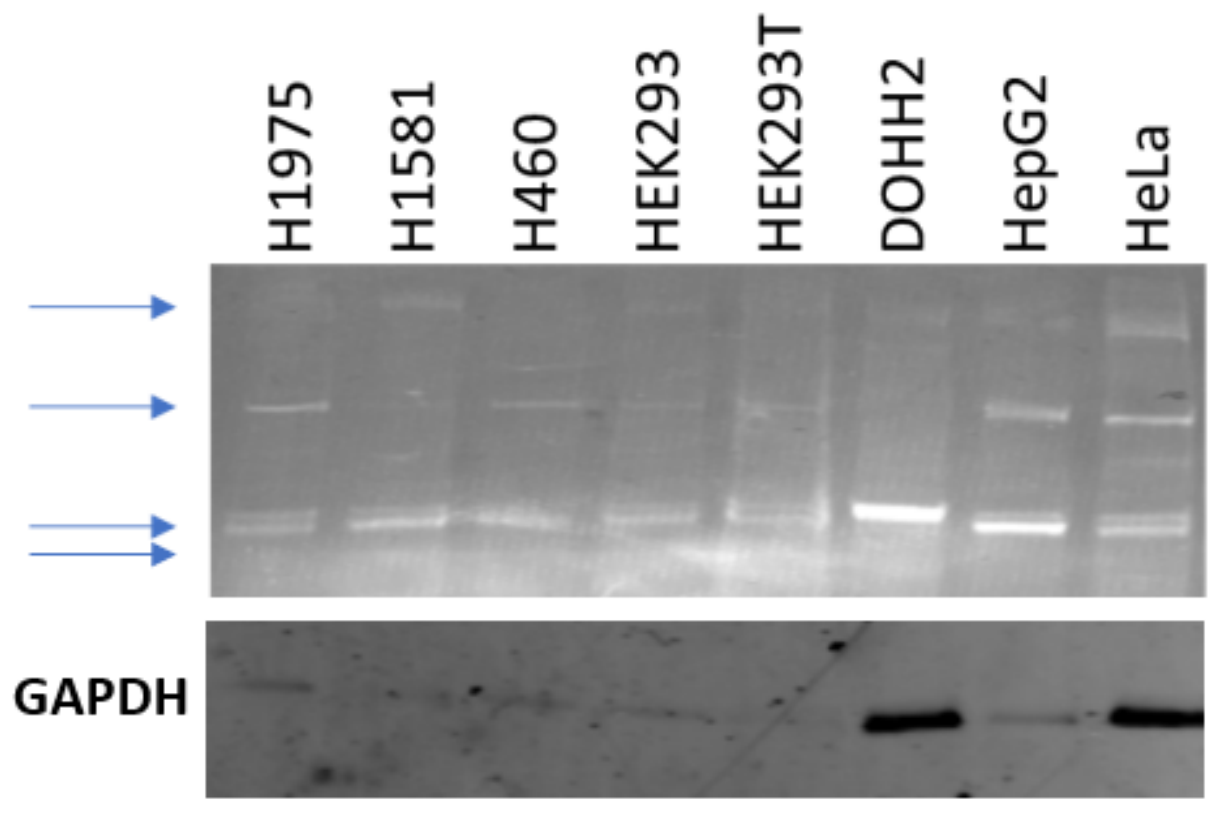

Figure 2

Expression of LPAR1 in human cell lines. 
a.

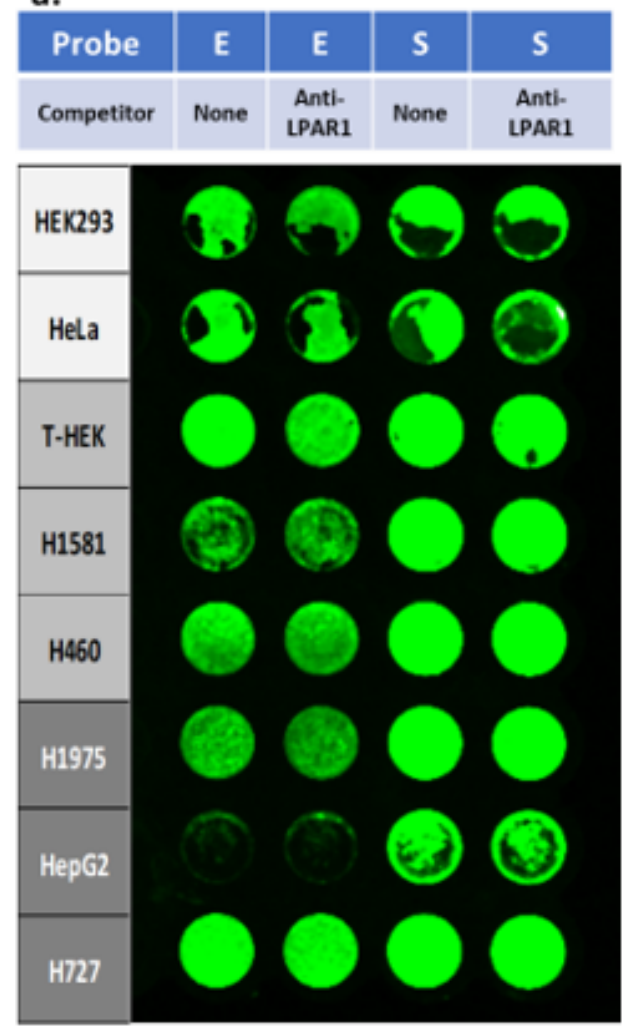

b.

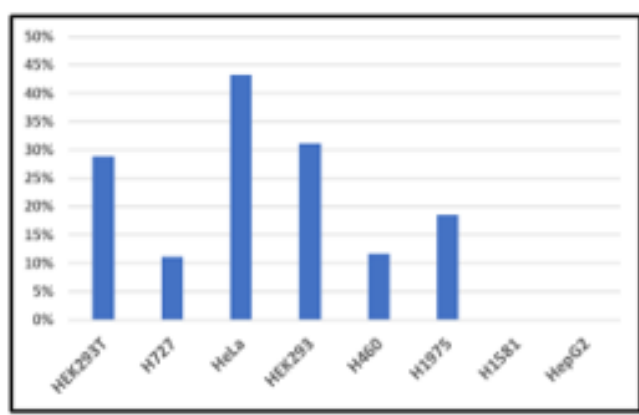

C.

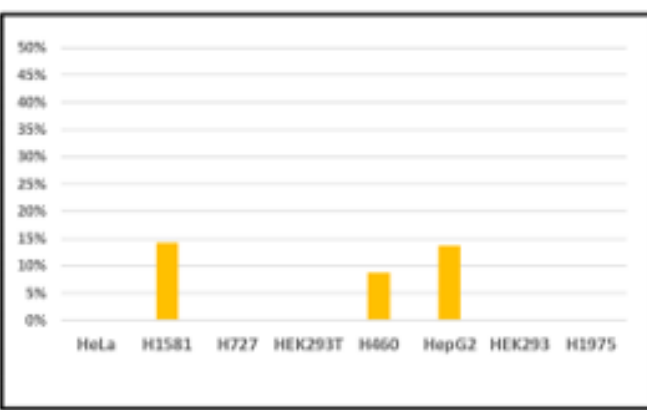

d.

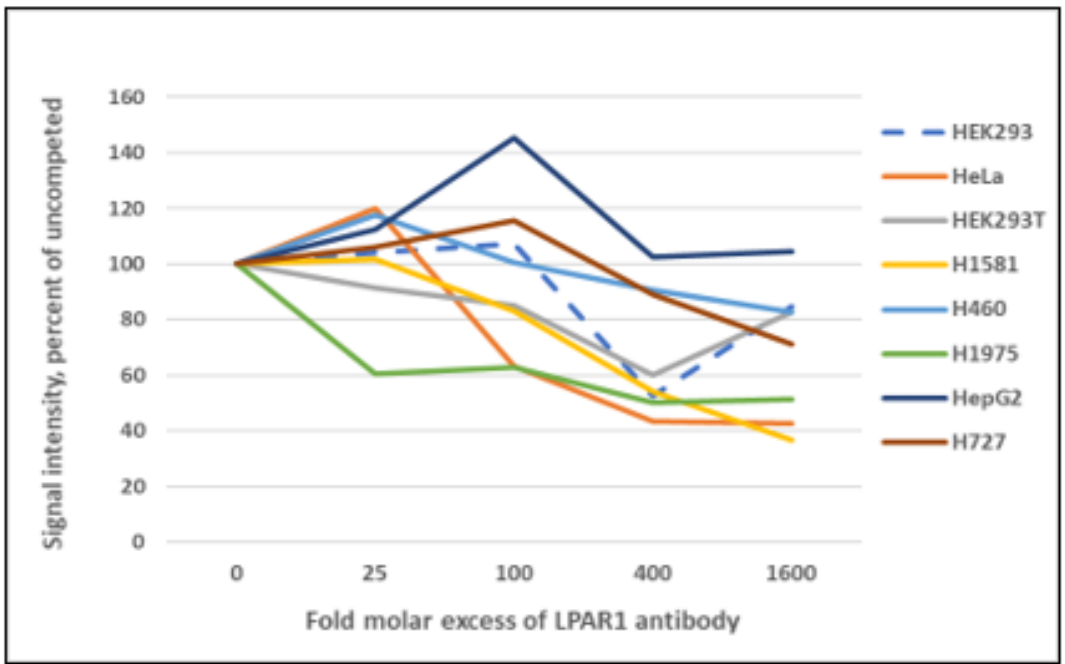

Figure 3

LPAR1 antibody inhibits binding of E protein to cells in vitro. 

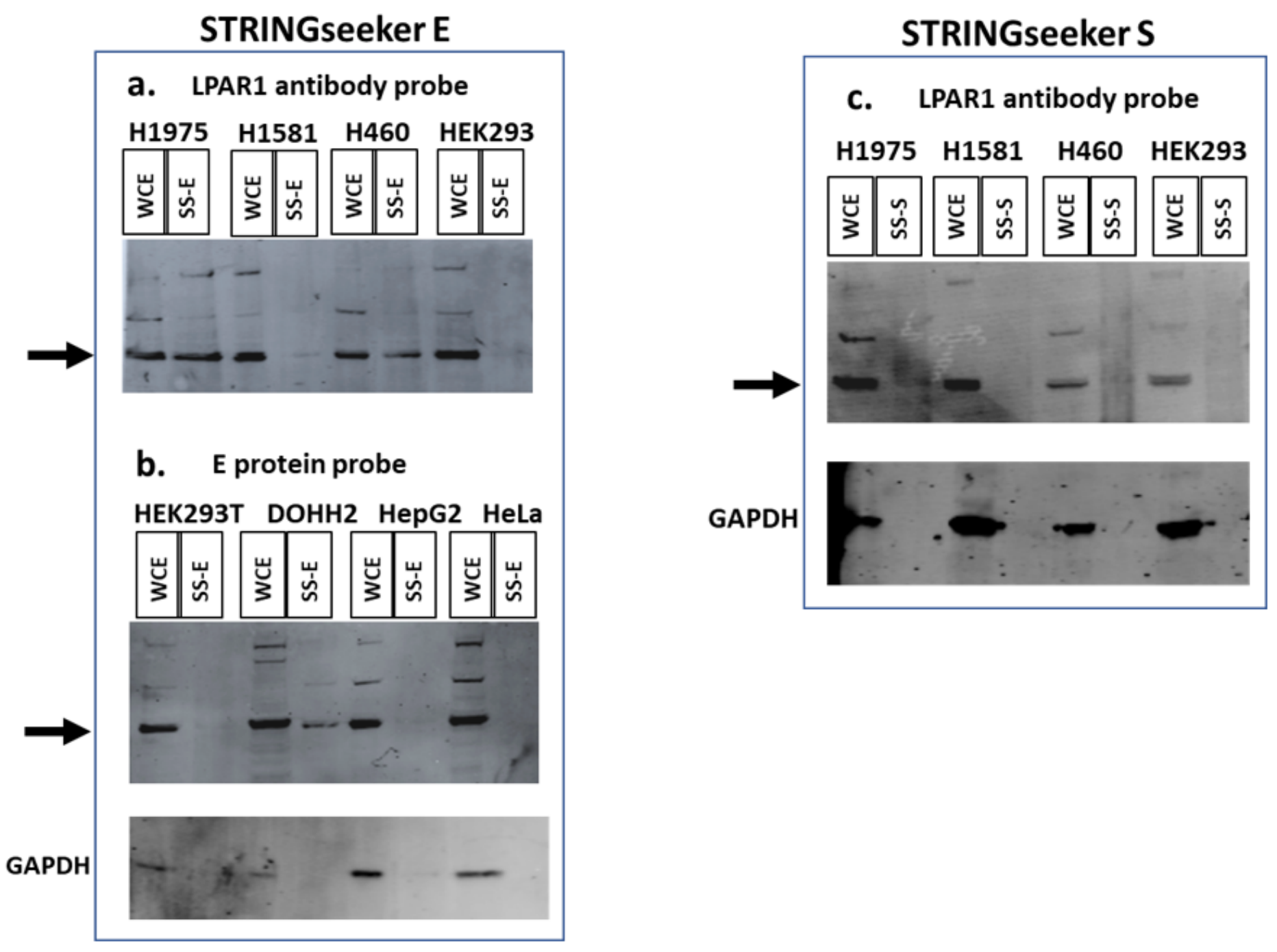

Figure 4

LPAR1 physically associates with the E protein in vitro and co-purifies with STRINGseeker products. 